\title{
Retraction Note to: Relationship of serum MMP-7 levels for colorectal cancer: a meta-analysis
}

\author{
Xiao-Jing Xing ${ }^{1} \cdot$ Xiao-Hu Gu ${ }^{2} \cdot$ Tian-Fei Ma ${ }^{1}$
}

Published online: 17 August 2015

(C) International Society of Oncology and BioMarkers (ISOBM) 2015

Retraction to: Tumor Biol. (2014) 35:10515-10522

DOI 10.1007/s13277-014-2349-3

The Publisher and Editor retract this article in accordance with the recommendations of the Committee on Publication Ethics (COPE). After a thorough investigation we have strong reason to believe that the peer review process was compromised.

The online version of the original article can be found at http://dx.doi.org/ 10.1007/s13277-014-2349-3.

Xiao-Jing Xing

xingxiaojian69@126.com

1 Department of Internal Oncology, Liaoning Cancer Hospital and Institute, No. 44, Xiaoheyan Road, Dadong District,

Shenyang 110042, Liaoning Province, People's Republic of China

2 Department of Surgical Oncology, Liaoning Cancer Hospital and Institute, Shenyang 110042, Liaoning Province, People's Republic of China 